\title{
Follow-up after EUS-guided radiofrequency ablation for pancreatic lesions: With more answers, come more questions
}

\section{다(1) $(-)$}

\author{
Authors \\ Ryan Law, Michael J. Levy \\ Institution \\ Mayo Clinic, Department of Gastroenterology and \\ Hepatology, Rochester, Minnesota, United States \\ Bibliography \\ Endosc Int Open 2021; 09: E1186-E1187 \\ DOI 10.1055/a-1393-5780 \\ ISSN 2364-3722 \\ (c) 2021. The Author(s).
}

\author{
This is an open access article published by Thieme under the terms of the Creative \\ Commons Attribution-NonDerivative-NonCommercial License, permitting copying \\ and reproduction so long as the original work is given appropriate credit. Contents \\ may not be used for commercial purposes, or adapted, remixed, transformed or \\ built upon. (https://creativecommons.org/licenses/by-nc-nd/4.0/) \\ Georg Thieme Verlag KG, Rüdigerstraße 14, \\ 70469 Stuttgart, Germany \\ Corresponding author \\ Ryan Law, DO, 200 1st, Street SW, Rochester, MN 55905, \\ United States \\ drryanlaw@gmail.com
}

Radiofrequency ablation (RFA) is a recently described technology for the treatment of low-grade pancreas lesions, such as neuroendocrine tumors (NETs) and pancreatic cystic neoplasms (PCNs). Early data suggest that this intervention is safe and effective; however, numerous questions remain regarding its role in our therapeutic armamentarium. RFA employs electromagnetic energy and high-frequency alternating currents to induce coagulative necrosis and fibrotic changes in highly thermosensitive tissues, such as the pancreas [1-3] This method of cell death causes the release of intracellular antigens known to stimulate a delayed immune response as well [4]. To date, a paucity of studies have examined the utility of RFA in treating pancreas lesions, and data on long-term outcomes are non-existent.

In 2015, one of the first multicenter studies to examine the efficacy of EUS-RFA ablation reported results from eight patients with pancreas lesions (4 mucinous cystic neoplasms (MCN), 1 intraductal papillary mucinous neoplasm [IPMN], 1 microcystic adenoma, 2 NET) [2]. Complete resolution was described in two patients with cystic lesions with a $48 \%$ volume reduction in three other patients with cystic lesions. No severe post-procedural adverse events occurred. In the NET patients, notable post-treatment effect was identified. It should be mentioned that this study utilized a probe-based EUS-RFA technology (Habib EUS-RFA catheter; Emcision, Ltd., London) that was passed through the lumen of an EUS-FNA needle.

More recently, the authors of the present study published their early data on 30 patients (14 NET, 16 branch duct IPMN, and $1 \mathrm{MCN}$ ) which aimed to further evaluate the safety profile of RFA [4] This study was performed using a newer iteration of EUS-RFA technology which utilizes an 18G RFA needle (EUSRA/ Starmed;Taewoong, South Korea) passed directly into the lesion of interest. During the 1 -year follow-up there was a $10 \%$ adverse event $(\mathrm{AE})$ rate, including two severe AEs (acute pancreatitis with infected pancreatic necrosis, jejunal perforation). Interestingly, these two severe AEs occurred in the initial two patients enrolled. The protocol was subsequently modified to include prophylaxis against acute pancreatitis and periprocedural antibiotics. No additional severe adverse events occurred in the remaining 28 patients. In terms of technical success, all lesions were treated successfully. At 6- and 12-month followup of patients with NETs, complete resolution was noted in $71 \%$ and $86 \%$ of the lesions, respectively. Among the 17 patients with PCNs, resolution was noted in $47 \%$ and $65 \%$ of lesions at 6- and 12-month follow-up, respectively. The delayed response in resolution is thought to occur secondary to local and systemic immune activation when intracellular contents are destroyed, released and intersected by dendritic cells [5]. These findings have raised important clinical questions regarding the time to full effect, as well as the risk of recurrence and duration of follow-up necessary after EUS-RFA of pancreas lesions.

In this issue of Endoscopy International Open, Barthet et al [6] report on the efficacy of EUS-RFA in the treatment of nonoperative patients with either a NET $<2 \mathrm{~cm}(n=12)$ or PCN $(n=$ 17) who were subsequently followed for $>3$ years after treatment (mean 43 months). Follow-up cross sectional imaging or EUS was used to determine the post-RFA results (i.e. complete 
resolution [disappearance of necrosis], significant response [decrease $>50 \%$ or complete resolution], or failure [decrease $<50 \%$ or no effect]. In the 12 patients with NETs, complete resolution was noted in $85 \%$ of patients at 1 year and at the end of the follow-up period; however, two failures were noted (1 patient with complete resolution at 1 year followed by recurrence, and 1 patient who failed therapy at 1 year and failed a second EUS-RFA treatment who subsequently developed metastatic disease). In patients with PCNs, $66 \%$ demonstrated a significant response during the follow-up period, with five patients meeting failure criteria. Interestingly, at 1 year, the significant response rate was $70 \%$, slightly higher than the results at 3 years (66\%). In the 10 patients with PCNs and a mural nodule, the nodule resolved completely with EUS-RFA in all cases, suggesting anecdotally a decreased risk for malignant progression going forward. Two patients in the PCN cohort were diagnosed with pancreatic ductal adenocarcinoma at a location distant to the cystic lesion treated with EUS-RFA. The overall AE rate was $13 \%$, all of which were periprocedural. No patients developed long-term sequelae or experienced delayed AEs related to EUSRFA.

We applaud the work and effort by Barthet and colleagues [6] designed to help shine light on the role and long-term efficacy of pancreatic RFA. The present manuscript has begun to shed light on long-term outcomes and initiated the discussion regarding potential surveillance strategies post-RFA. We believe their work also highlights the many questions we, and all endosonographers and clinicians, have when contemplating the use of this technology, namely:

1. Defining success: What should be the clinical and imaging parameters of therapeutic success? The presumed development of necrosis, scar formation, alterations of enhancement, decreases in size, etc. As shown in the patients who developed liver metastasis, did this represent prior occult metastasis that required additional growth to allow detection and/or residual tumor in an insufficiently treated tumor? More concerning, is the patient with complete resolution at 1 -year with recurrence at the 3 -year follow-up. Are we simply leaving a prettier lesion that remains at risk?

2. Defining long-term follow-up: Given the typical slow growth of NETs and PCNs, a 3-year follow-up may simply allow a general assessment of perceived adequacy of ablation, but not allow a meaningful determination of true clinical success and improved outcomes. In addition, the length of follow-up post-RFA may be different based on the type of lesion. Should PCNs and NETs be surveilled in the same manner?

3. Proper risk tolerance: Risk tolerance must be balanced against therapeutic benefit. The rate and notable AEs reported in this study highlight the tenuous role of RFA.
4. Impact on surveillance imaging: Given the uncertainties defining and verifying treatment success, especially at a cytohistological level, it is unclear whether surveillance imaging can be abandoned in such patients; and finally,

5. Patient selection: Among the 12 patients reported, four died of unrelated causes, another developed liver metastasis, and one had co-existing pancreatic ductal adenocarcinoma with treatment of a remote cyst. Given the slow-growing nature of most of the treated lesions, and all of the aforementioned questions, more thought and consensus is needed to better define the target population.

To date, the majority of published data have provided limited insight into the long-term outcomes of patients undergoing EUS-RFA for small pancreas lesions. The data presented Barthet and colleagues [6] are currently the best available evidence regarding long-term outcomes of such cases, and they should be commended. Our comments should not be viewed as unfavorable toward the investigators, their work, or this technology in any way. We, too, perform EUS-RFA and very much struggle with each of these questions and uncertainties and eagerly await subsequent data to provide clarity.

\section{Competing interests}

The authors declare that they have no conflict of interest.

\section{References}

[1] Chaudhary S, Sun SY. Endoscopic ultrasound-guided radiofrequency ablation in gastroenterology: New horizons in search. World J Gastroenterol 2017; 23: 4892-4896

[2] Pai M, Habib N, Senturk $\mathrm{H}$ et al. Endoscopic ultrasound guided radiofrequency ablation, for pancreatic cystic neoplasms and neuroendocrine tumors. World J Gastrointest Surg 2015; 7: 52-59

[3] Lakhtakia S, Seo DW. Endoscopic ultrasonography-guided tumor ablation. Dig Endosc 2017; 29: 486-494

[4] Barthet M, Giovannini M, Lesavre N et al. Endoscopic ultrasoundguided radiofrequency ablation for pancreatic neuroendocrine tumors and pancreatic cystic neoplasms: a prospective multicenter study. Endoscopy 2019; 51: 836-842

[5] Slovak R, Ludwig JM, Gettinger SN et al. Immuno-thermal ablations boosting the anticancer immune response. J Immunother Cancer 2017; 5: 78

[6] Barthet M. Long-term outcome after EUS-guided radiofrequency ablation: Prospective results in pancreatic neuroendocrine tumor and pancreatic cystic neoplasms. Endoscopy International Open 2020; 09: E1178-E1185 